\title{
Coercivity dynamics in micromagnets *
}

\author{
Huei-Li Huang, Ivo Klik, Ching-Ray Chang and Kuo-Kang Liang \\ Department of Physics, National Taiwan University, Taipei, Taiwan
}

\begin{abstract}
For a system of non-interacting uniaxial micromagnmetic particles we calculate the temperature dependent dynamic coercivity for sweep rates $f=10^{-1}-10^{8} \mathrm{~Hz}$ and for a wide range of temperatures. The coercivity is found numericaly from an analytic expression for the hysteresis loop. Thermal activation and backscattering are taken into account.
\end{abstract}

The hysteresis properties of particulate media have attracted a great deal of attention, see e.g. ref. [1], due to their importance to high density recording. Similar attention is recently being drawn to granular magnetic materials [2]. Of particular interest, in view of the currently used short access times, is the frequency (and temperature) dependence of the coercive field - a problem of high complexity usually treated phenomenologically or by means of computer simulation. The underlying mechanism is, of course, well understood, but difficulties arise in determining the instanteneous state of a system responding to time varying magnetic field. Based on a reduced Fokker-Planck equation we developed [3] a formula for the non-equilibrium probability distribution which propagates an arbitrary initial state in time and we use it to calculate the non-equilibrium magnetization as a function of the instantaneous applied magnetic field.

The article is arranged as follows: first we present our basic theory and proceed then to apply it to a model uniaxial system with easy axis in the direction of the applied field. We give a plot of the dynamic, temperature dependent coercivity and give a brief description of our ongoing work which aims at analytic description of a realistic system of (non-interacting) particles.

We base our analysis of the dynamic response of micromagnets to time varying magnetic field $B(t)$ on the equation [3]

$$
\begin{aligned}
\hat{P}\left(T \mid B, B_{0}\right)= & P(T, B)+\left[P_{\text {in }}-P\left(T, B_{0}\right)\right] \mathrm{e}^{-\epsilon\left(B, B_{0}\right)} \\
& -\int_{B_{0}}^{B} \mathrm{~d} B^{\prime} \frac{\partial P\left(T, B^{\prime}\right)}{\partial B^{\prime}} \mathrm{e}^{-\epsilon\left(B^{\prime}, B_{0}\right)}
\end{aligned}
$$

The temperature $T$ is assumed to be constant and $k_{\mathrm{B}} \equiv 1$. In eq. (1) $P(T, B)$ is the normalized thermal

Correspondence to: Dr. Huei-Li Huang, Department of Physics, National Taiwan University, Taipei 10764, Taiwan.

* This research was supported by a National Science Council grant number NSC-81-0405-E002-13. equilibrium distribution for which the relation $\partial P / \partial B$ $=T^{-1}\left(\langle\partial E / \partial B\rangle_{B}-\partial E / \partial B\right) P$ proves to be useful in calculations; $E$ is the magnetic energy and $\langle x\rangle_{B}=$ $\operatorname{Tr} x P(T, B)$ the equilibrium value of $x$. The initial distribution at field $B_{0}$ is usually taken as $P_{\text {in }} \equiv$ $P\left(T, B_{0}\right)$. The function $\hat{P}\left(B, B_{0}\right)$ gives the normalized non-equilibrium distribution of a system subject to time varying external magnetic field $B(T)$ with initial value $B_{0}=B\left(t_{0}\right)$. Finally, the relaxation exponent $\epsilon$ is defined as

$$
\begin{aligned}
\epsilon\left(B, B_{0}\right) & =\int_{t_{0}}^{t} \mathrm{~d} t^{\prime} \Gamma\left[T, B\left(t^{\prime}\right)\right] \\
& =\int_{B_{0}}^{B} \mathrm{~d} B^{\prime} \frac{\partial t\left(B^{\prime}\right)}{\partial B^{\prime}} \Gamma\left[T, B^{\prime}\right],
\end{aligned}
$$

where $t(B)$ is the inverse of $B(t)$. The function $\Gamma[T, B]$ is the net rate of relaxation towards thermal equilibrium. For our system of uniaxial (two well) non-interacting particles, see eq. (5) below, we write it as the net flux rate through the saddle point separating the two wells

$$
\begin{aligned}
\Gamma[T, B]= & n_{1}\left(B, B_{0}\right) f_{0} \exp \left[-Q_{1 \rightarrow 2} / T\right] \\
& -n_{2}\left(B, B_{0}\right) f_{0} \exp \left[-Q_{2 \rightarrow 1} / T\right] .
\end{aligned}
$$

Here $Q_{1 \rightarrow 2}$ is the barrier height on transition from well 1 to 2 and similarly for $Q_{2 \rightarrow 1}$. We estimate the prefactor $f_{0}$ as $\mathrm{e}^{25} \mathrm{~Hz}$. The instanteneous population in well 1 is given by the integral $n_{1}\left(B, B_{0}\right)=$ $\int_{A_{1}} \mathrm{~d} A \hat{P}\left(B, B_{0}\right)$ over the domain of attraction $A_{1}$ of the first well [4]. Obviously $n_{1}+n_{2} \equiv 1$ at all times since the two domains of attraction $A_{1}$ and $A_{2}$ cover the entire phase space and a decrease in $n_{i}$ is reflected in decreased flux out of the $i$ th well by definition of the prefactor.

The expression (3) takes into account both the effects of backscattering and of the variation in the number of particles at the saddle point energy [4]. We find an interesting property of the rate function $\Gamma[T, B(t)]$ : it has a maximum close to the dynamic 
coercive field $B_{\mathrm{c}}$ while at higher fields $\Gamma \rightarrow 0$ as opposed to the thermal decay rate which increases rapidly as $B$ approaches the nucleation field $B_{n}$. This is easily understood since at $B_{\mathrm{n}}>B>B_{\mathrm{c}}$ the transition probability $1 \rightarrow 2$ is large but the population of well 1 is already strongly depleted. Also, this process is countered by the reverse flux $2 \rightarrow 1$ which has low transition probability, $Q_{1 \rightarrow 2}>Q_{2 \rightarrow 1}$, but which originates in the richly populated well 2 . The calculated hysteresis curve is steepest close to $B_{\mathrm{c}}$ (specifically at fields somewhat larger than $B_{\mathrm{c}}$, but this deviation is noticeable only at high temperatures) and it exhibits a long tail on approach to the equilibrium (Langevin) curve where the net relaxation rate significantly decreases.

Given the above theory it is easy to determine the non-equilibrium magnetization

$$
\begin{aligned}
\hat{M}\left(T \mid B, B_{0}\right)= & \operatorname{Tr} M \hat{P}\left(B, B_{0}\right) \\
= & \langle M\rangle_{B}+\left[\langle M\rangle_{\mathrm{in}}-\langle M\rangle_{B_{0}}\right] \mathrm{e}^{-\epsilon\left(B, B_{0}\right)} \\
& -\int_{B_{0}}^{B} \mathrm{~d} B^{\prime} \frac{\partial\langle M\rangle_{B^{\prime}}}{\partial B^{\prime}} \mathrm{e}^{-\epsilon\left(B^{\prime}, B_{0}\right)}
\end{aligned}
$$

The derivative may conveniently be calculated from the general formula $\partial\langle M\rangle_{B} / \partial B=T^{-1}\left[\langle\partial E / \partial B\rangle_{B}\langle M\rangle_{B}-\right.$ $\left.\langle M(\partial E / \partial B)\rangle_{B}\right]$.

In our model calculation we assume that the magnetic energy is given by

$E=v\left[-B M_{\mathrm{s}} p+K\left(1-p^{2}\right)\right] \quad p=\cos \theta$,

where $v$ is activation volume, $M_{\mathrm{s}}$ the saturation magnetization, $K$ the anisotropy constant and we set $B=$ $B(t)=B_{\mathrm{n}} \cos (2 \pi f t)$. The system, initially in thermal equilibrium at $B=-B_{\mathrm{n}}=-2 K / M_{\mathrm{s}}$ (i.e. spin down, $P_{\text {in }}=P\left(T,-B_{\mathrm{n}}\right)$ ), is driven by magnetic field of frequency $f$. Any other loop can be calculated with a suitable choice of initial conditions; to the virgin curve there corresponds $P_{\text {in }}=P(T, 0)$. Let further $b=B / B_{\mathrm{n}}$ $\in\langle-1,1\rangle$ so that $Q_{1 \rightarrow 2}=K v(1+b)^{2}$ and $Q_{2 \rightarrow 1}=$ $K v(1-b)^{2}$. For magnetic field in the 3-direction we obtain the simple relations $\partial\langle p\rangle_{B} / \partial b=-2 K v T^{-1}\langle(p$ $\left.\left.-\langle p\rangle_{B}\right)^{2}\right\rangle_{B}, \partial P / \partial b=2 Q\left(\langle p\rangle_{B}-p\right) P$ which hold for arbitrary anisotropy energy. The thermal averages are conveniently calculated numerically or, indeed, as Gaussian integrals in the asymptotic limit $K v / T \gg 1$.

From eq. (4) we have calculated hysteresis loops for a wide range of frequencies, $f=10^{-1}-10^{8} \mathrm{~Hz}$, and temperatures, $K v / T=10-260$, and obtained a number of hysteresis loops. The coercive field $B_{\mathrm{c}}(f, T)$ is plotted in fig. $1, B_{c}(f)_{T=\text { const }}$, and fig. $2, B_{c}(T)_{f=\text { const }}$. We have not attempted to fit the data to any particular experimental curve - we discuss this problem in the concluding paragraph - but sought rather to calculate

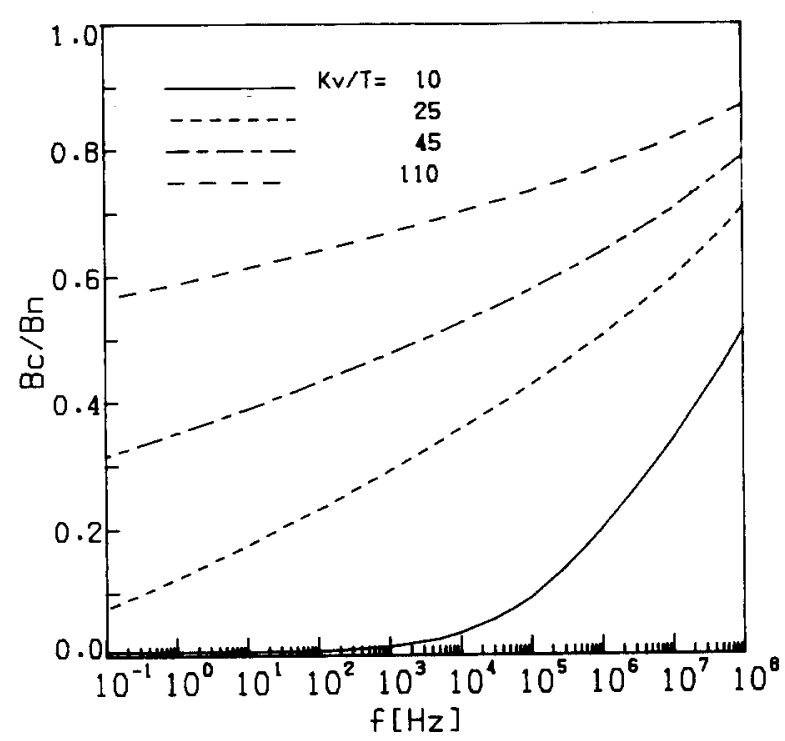

Fig. 1. The reduced dynamic coercive field $B_{\mathrm{c}} / B_{\mathrm{n}}$ at constant temperature as a function of the sweep rate $f$.

the function $B_{\mathrm{c}}(f, T)$ in the whole range of physical interest. The most prominent feature of $B_{c}(f, T)$ is the transition from the prefactor dominated $(K v / T<25)$ to the Arhhenius factor dominated behaviour at low temperatures $(K v / T>25)$. We see that for high temperatures $B_{c}(f)$ is very close to zero even for moderately large $f$ and increases then steeply towards saturation. On the other hand, at low temperatures $B_{c}$ is appreciably greater than zero even at $f=10^{-1} \mathrm{~Hz}$, but

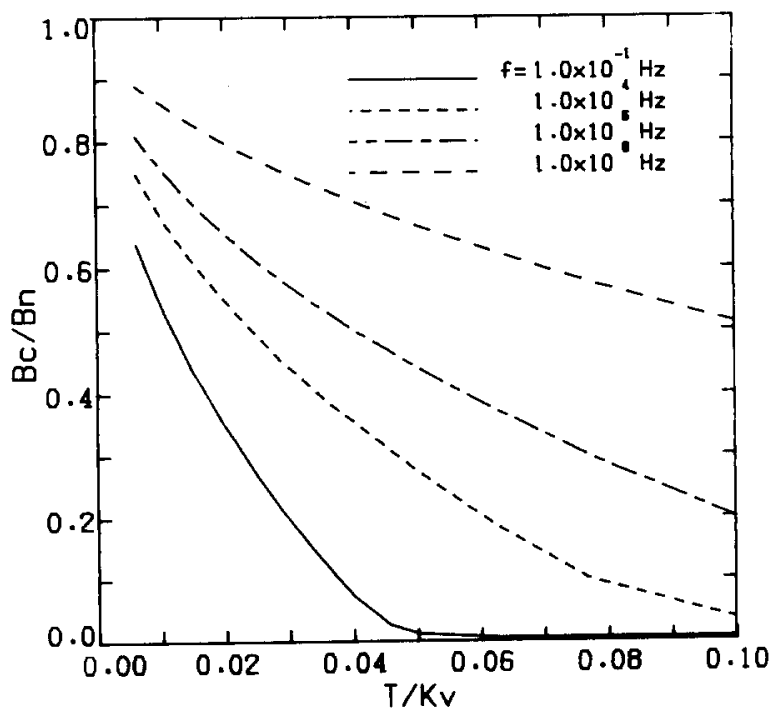

Fig. 2. The reduced dynamic coercive field $B_{\mathrm{c}} / B_{\mathrm{n}}$ at constant frequency as a function of the reduced temperature $T /(K v)$. 
the $f$-dependence is far less pronounced. The transition is equally well discernible in the plot of $B_{c}(T)$ at constant frequency, fig. 2 , especially at small $f$. Saturation of $B_{\mathrm{c}}$ occurs only at $f \approx f_{0}$, i.e. outside of the region of practical interest. Let it yet be remarked that the choice of $f_{0}=\mathrm{e}^{25} \mathrm{~Hz}$ results in no loss of generality: from eq. (2) it follows that $\epsilon \propto f_{0} / f$ so that a change in $f_{0}$ results merely in a shift of the plots 1 and 2.

Our system of identical particles exactly aligned with the field is not realistic, nor is the trivial generalization of eq. (5) in which the easy axis is rotated by an angle $\psi$ from the field direction so that $B_{\mathrm{c}}=$ $B_{c}(f, T, \psi)$. The definition (3) guarantees that $\lim _{\psi \rightarrow \pi / 2} B_{c}(f, T, \psi)=0$ as should be. In a real system the activation volume $v$ has a log-normal and the angle $\psi$ a " $\cos ^{4} "$ distribution. The mean magnetization is found by averaging the function $\hat{M}\left(B, B_{0}\right)$, the req- uisite double integral is conveniently evaluated using Gauss-Legendre and Gaussian quadratures respectively. We shall report on our results in near future. The present analytic treatment allows to include consistently such effects as the temperature dependence of the magnetic parameters and prefactor $[3,4]$, the dynamic dependence of the activation volume [5] etc., and is therefore eminently suitable to the study of non-interacting systems.

\section{References}

[1] R.W. Chantrell, J. Magn. Magn. Mater. 95 (1991) 365.

[2] C.L. Chien, J. Appl. Phys. 69 (1991) 5267.

[3] I. Klik, C.-R. Chang and H.-L. Huang, Phys. Rev. B, submitted.

[4] I. Klik and L. Gunther, J. Stat. Phys. 60 (1990) 473.

[5] M.E. Schabes, J. Magn. Magn. Mater. 95 (1991) 249. 